\title{
EVALUATION OF MODEL RECOGNITION FOR GRAMMAR-BASED AUTOMATIC 3D BUILDING MODEL RECONSTRUCTION
}

\author{
Qian Yu, Petra Helmholz and David Belton \\ Cooperative Research Centre for Spatial Sciences (CRCSI) \\ Department of Spatial Sciences, Curtin University \\ GPO Box U 1987, Perth, WA, 6845, Australia \\ qian.yu9@postgrad.curtin.edu.au, (petra.helmholz, d.belton)@ curtin.edu.au
}

Commission IV, WG IV/1

KEY WORDS: building model, evaluation, reconstruction, 3D, grammar

\begin{abstract}
:
In recent years, 3D city models are in high demand by many public and private organisations, and the steadily growing capacity in both quality and quantity are increasing demand. The quality evaluation of these $3 \mathrm{D}$ models is a relevant issue both from the scientific and practical points of view. In this paper, we present a method for the quality evaluation of 3D building models which are reconstructed automatically from terrestrial laser scanning (TLS) data based on an attributed building grammar. The entire evaluation process has been performed in all the three dimensions in terms of completeness and correctness of the reconstruction. Six quality measures are introduced to apply on four datasets of reconstructed building models in order to describe the quality of the automatic reconstruction, and also are assessed on their validity from the evaluation point of view.
\end{abstract}

\section{INTRODUCTION}

In the recent decade modelling and 3D description of real world objects has become a topic of increasing importance as they are essential for a variety of applications among different fields such as urban planning, construction, environment safety, navigation, and virtual city tourism (Haala and Kada, 2010). Due to the increasing demands in both quality and quantity of $3 \mathrm{D}$ models within those fields, automatic reconstruction is essential for the acquisition and updating of the huge amount of data to avoid the labour intensive and time consuming manual processing work flow (Helmholz et al., 2013).

Compared with traditional digital imagery, nowadays terrestrial laser scanning provides explicit 3D information, which enables the possibility to rapid and accurately capture the geometry of complex buildings. Much work has been already done on the semi-automatic or automatic approaches for building reconstruction from terrestrial laser scanning data, especially using grammar-based methods (Brenner, 2005; Milde et al., 2008). However, in contrast to the huge amount of literature dealing with the method for building reconstruction, there are only a few references focusing on evaluation of the reconstructed building models (Akca et al., 2010). Evaluation process plays a significant role in building model reconstruction since it may give important information about deficiencies of a reconstruction approach and help improve the approach. Furthermore, the quality of a reconstructed model can be checked not only based on visual inspection which is subjective, but also on quantitative measures.

Therefore, in this paper, we present an evaluation approach for our grammar-based automatic 3D building model reconstruction method. In order to evaluate the building models in professional practice, we have introduced a few quantitative quality measures to reflect the evaluation results from both building model completeness and building model correctness aspects. The rest of the paper is organised as follows. In Section 2, the

\footnotetext{
${ }^{*}$ Corresponding author
}

background related to our work is introduced. In Section 3, we briefly describe the way to reconstruct a building model used our grammar-based method, then the proposed evaluation approach for the reconstructed model is presented in detail. In Section 4, we show the evaluation results of two reconstructed building models. Finally, conclusions are discussed in Section 5.

\section{RELATED WORK}

Much research concerning grammar-based methods used in architecture modelling has been published. The most well-known examples are the L-systems, which have been used for extracting the buildings and streets from aerial imagery by Parish and Müller (2001). Then the idea of shape grammars was originally introduced by Stiny and Gips (1972), and successfully used in architecture by McKay et al. (2012). In 2003, Wonka et al. (2003) employed a split grammar to generate architectural structures based on a large database of split grammar rules and attributes. Following this idea, a new Computer Generated Architecture (CGA) grammar (Müller et al., 2006) has been developed to generate detailed building architecture in a predefined style. More recently, formal grammars have been applied to reconstruct building façades (Becker, 2009; Becker and Haala, 2009) from terrestrial laser scanning data. In order to reconstruct full building structures automatically, our group also have proposed a method for automatic building reconstruction using an attributed building grammar (Yu et al., 2014).

While the performance of the grammar-based methods is improving, the quality evaluation of building models has become an important issue. A number of papers were published focusing on calculating through the metrics using pixels based on $2 \mathrm{D}$ projections. In 1997, Henricsson and Baltsavias (1997) has presented a qualitative and quantitative evaluation of the reconstructed $3 \mathrm{D}$ roofs relative to accurate reference data. However, as the reference data is $2 \mathrm{D}$ aerial images, it needs to project a $3 \mathrm{D}$ object onto a $2 \mathrm{D}$ plane for analysing the differences. Later, a concept of Feature Based Model Verification (FBMV) has been proposed (Ameri, 2000), which 


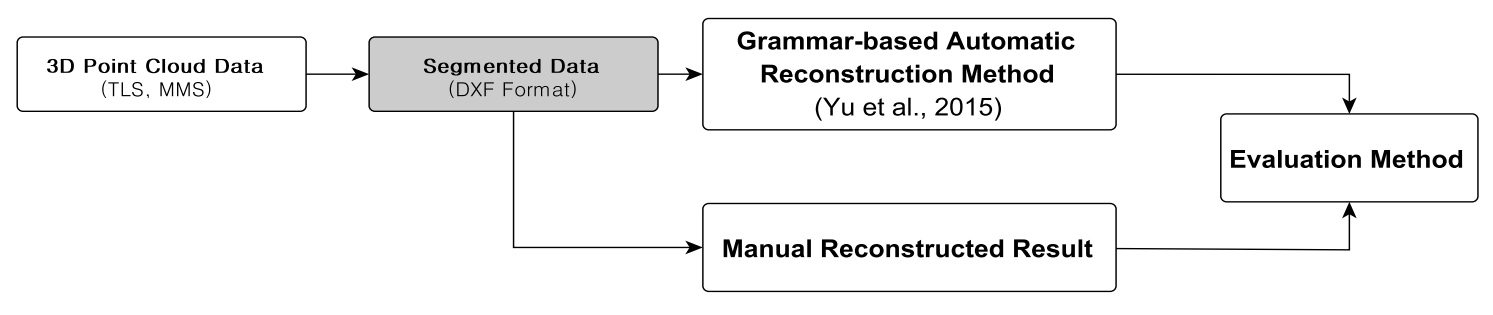

Figure 1: Proposed evaluation method using model attributes for automatic 3D building reconstruction.

aims to develop an automated method for recognition and 3D reconstruction of generic building objects using aerial images. The verification process is performed by fitting the reconstructed model primitives into the $2 \mathrm{D}$ features in images taken from different viewpoints, which is still based on 2D projection. A Similar evaluation method that compares 3D object models to images can be found in Suveg and Vosselman (2002). After publishing a grammar-based façade reconstruction method, in 2009, Ripperda and Brenner (2009) has evaluated this reconstruction method with façade images, which examines the reconstructions pixel based.

In addition, many papers evaluate building models using voxels by considering buildings as volumetric data. Volume metrics are used in building model evaluation to compare the reconstructed data for each voxel to the reference data (McKeown et al., 2000), which is an extension on the work of McGlone and Shufelt (1994). However, the volume metrics are affected by building size, which results in the method working well on large buildings, but not small buildings. In 2003, Schuster and Weidner (2003) have extended the work of McKeown et al. (2000), and presented an approach for the evaluation of 3D building models. The evaluation process can be able to decided by the user to evaluate the full $3 \mathrm{D}$ geometry, the $2 \mathrm{D}$ positional geometry or the height. Nonetheless, the building models were compiled from aerial images, and the evaluation result presented in the paper is actually based on 2D information only.

Besides the methods based on pixels and voxels, qualitative and visual evaluation based methods are also preferred. Visual evaluations were performed by comparison test data sets from semi-automatic building reconstruction systems to reference data sets from aerial images (Rottensteiner and Schulze, 2003; Durupt and Taillandier, 2006). Moreira et al. (2013) have presented a quantitative evaluation approach for 3D building models generated from LiDAR data. In their approach, an observation was carried out to compare selected building models with the real dimensions of actually surveyed buildings to assess the quality of the generated building models. However, as the reconstructed models are Level of Detail (LoD)2 building models (Biljecki et al., 2013), only topological structures is observed in the evaluation process.

Since the 3D building models reconstructed from the grammar-based method are in solid model form (LoD3), pixel or voxel representations are not suitable for quality evaluation of these models. Thus, in this paper, we propose an evaluation method, which directly works on 3D solid elements (building parts). In order to evaluate the building model in a semantic way, a structurally rich description of a model is composed of a small number of 3D geometric primitives which have been interpreted as different building elements and contain the semantic information. The entire evaluation process can be divided into two parts, which are an evaluation of the building completeness, and an evaluation of the building correctness, respectively. For the sake of building completeness evaluation, well known quality measures, like e.g. detection rate, quality rate, miss factor, and false alarm rate. Meanwhile, the evaluation of building correctness mainly focuses on the aspects from semantic building structure, building elements, and their positions.

\section{METHODOLOGY}

\subsection{System Overview}

As shown in Figure 1, raw 3D point cloud data is processed in two independent ways to generate the 3D building models. One is to apply the grammar-based automatic reconstruction method to generate a test model (Yu et al., 2015). The other way is to manually build the 3D model with commercial software tools, e.g. Cyclone, which is used as a reference model. Our proposed evaluation method is to evaluate the completeness and correctness of a test model compared with a reference model. It is noted that the method used for evaluation can significant produce different "calculated values" even on the same data set from different reconstructed results. Therefore, without evaluation in a quantity manner, wrong impressions about the quality are possible only based on the objective judgement.

Before the detailed discussion with evaluation method, grammarbased automatic building model reconstruction method is briefly presented in the next subsection.

\subsection{Grammar-based Automatic Model Reconstruction}

The building model is reconstructed from segmentation data based on a building grammar. The segmentation data can be extracted from terrestrial laser scanning data in the pre-processing stage (Nurunnabi et al., 2012). As shown in Figure 2, the entire reconstruction process has been decomposed into two individual steps in order to reduce the overall complexity. In stage one, a structured boundary model is preliminarily derived from a polygonal model through the triangular mesh merging method similar to that presented by Rabin (2002), which explores the coherently structured 3D geometry and boundaries. The resulting building model should include additional geometric topological relations and appearance information. However, there is not much semantic information contained in such a structured boundary model. Thus, in stage two, a semantically structured model is further built based on an attributed building grammar and predefined rules from the intermediate results from the previous stage. The semantic model interprets the geometry structures with building information, which is determined in an automatic grammar-based reconstruction process. Within the semantic model, the semantic components e.g. walls, windows, doors and etc., could be linked with their geometry counterparts coherently. Details of building grammar and reconstruction method are given in (Yu et al., 2015). 


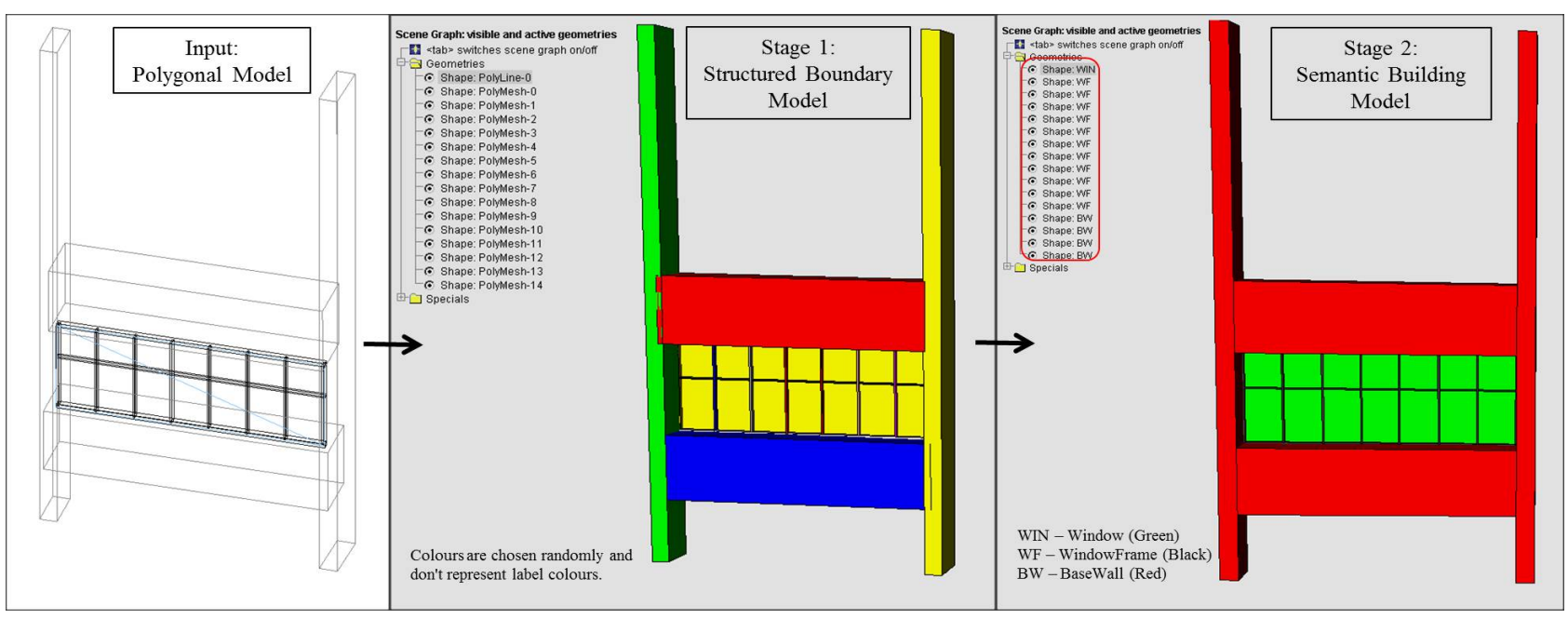

Figure 2: Diagram of grammar-based automatic building model reconstruction process.

\subsection{Quality Evaluation Process}

From Figure 3, there are three steps within the entire quality evaluation process according to the proposed grammar-based automatic building model reconstruction method, which includes the quality evaluation of the input terrestrial laser scanning (TLS) data, the segmentation data and the building model.

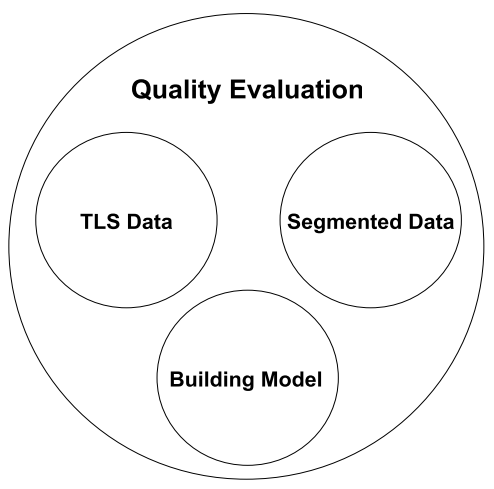

Figure 3: Diagram of quality evaluation process.

3.3.1 Quality Evaluation of TLS Data Generally, there are two important elements of the quality of raw terrestrial laser scanning data which are precision of TLS data and point density of the TLS data (Elberink and Vosselman, 2011). In term of precision of TLS data, systematic and stochastic errors could occur when acquiring the raw TLS data due to the measurement inaccuracies. In the other aspect, the point density plays important role to detect the refined objects from TLS data in the post-processing stage. In our case, we consider the quality of input TLS data is $6 \mathrm{~mm}$ (Leica C10) and point spacing is approximately $10 \mathrm{~mm}$, and therefore good enough to bring the negligible errors to last building model. More information as to the quality of TLS data is given in (Vosselman and Maas, 2010).

3.3.2 Quality Evaluation of Segmentation Data The quality of segmentation data is usually relation to the errors of missing segments caused by missing laser pulse returns, over-segmentation due to the fact that the growing radius locally is just too small, and under-segmentation caused by the appearance of laser points on two or more objects such that they seem to belong to the same segment. A more detailed description of segmentation errors is given in (Dorninger and
Pfeifer, 2008; Elberink and Vosselman, 2009). In this paper, both automatic model reconstruction method and manual processing method are using the same segmentation data set. As the building model from manual processing method is deemed as a reference model, the quality evaluation of segmentation data is outside of the scope of this paper.

3.3.3 Quality Evaluation of Building Model In order to evaluate the building model from the grammar-based automatic reconstruction method, there are two quality criteria addressed as follows, the correctness and the completeness.

In the context of this paper, the correctness mainly refers to the semantic information extracted from the building model elements. For example, the same build model element needs to have the consistent semantic attribute information i.e. window, door, wall and etc., no matter which reconstruction method is used. Due to the common segmentation data set applied for both manual and automatic methods, there is no need to further compare the geometry accuracy in term of positions and rotations. Comparision with a reference data is thereby limited within the high level interpretation of a 3D building model.

Another quality criterion is the completeness of a building model. It is important to evaluate the entire reconstructed building model not having the missing features or building parts compared with the verification building model. Assuming the verification data set is complete and accuracy, the missing detection rate will reflect the completeness of the building model. Currently there is no practical way to check fully automatically for this deficiency, and we will evaluate building completeness through the visual inspections and then calculate the missing rate.

To meet the two criteria above, a few quality measures are defined in 3.4 and applied in the test datasets.

\subsection{Quality Measures}

There exist a number of quality measures listed in the literature (Ragia, 2000; Schuster and Weidner, 2003). These have been derived for evaluating the quality of building models and can partially be converted in each other. Considering that the quality measures should be reliable when computed locally and easily be interpreted, a few quality measures are chosen, which are listed in the following. The values are computed based on the test data set $D$ and the reference data set $R$. 
- the detection rate

$$
\rho_{d}=\frac{|R \cap D|}{|R \cap D|+|R \backslash D|}=\frac{|R \cap D|}{|R|}, \rho_{d} \in[0,1]
$$

represents the rate of objects that are reconstructed only in the reference data set in relation to the number of reference objects.

- the branch factor

$$
\rho_{b}=\frac{|D \backslash R|}{|R \cap D|}, \rho_{b} \geq 0
$$

shows the rate of objects falsely reconstructed. Its optimum is zero.

- the quality rate

$$
\rho_{q}=\frac{|R \cap D|}{|R \cup D|}=1-\frac{|(D \backslash R) \cup(R \backslash D)|}{|R \cup D|}, \rho_{q} \in[0,1]
$$

The value of the quality rate is independent of the assignment of the reconstructed and reference data set. Its optimum is one.

- the miss factor

$$
\rho_{m}=\frac{|R \backslash D|}{|R \cap D|}, \rho_{m} \geq 0
$$

reveals the rate of objects not acquired. Its optimum is zero.

- the false alarm rate

$$
\rho_{f}=\frac{|D \backslash R|}{|R|}, \rho_{f} \geq 0
$$

represents the rate of the object that are not reconstructed in the reference data set in relation to the number of objects to be evaluated.

- the type 2 error

$$
\beta=\frac{|R \backslash D|}{|R|}, \beta \in[0,1]
$$

reveals the rate of the buildings or building parts not reconstructed. Its optimum is zero.

\section{RESULTS AND DISCUSSIONS}

In order to evaluate the results from grammar-based automatic building model reconstruction method, four datasets are evaluated and analysed with six introduced quality measures in this section. These datasets are listed as follows.

- Dataset 1: a single-floor building model reconstructed based on Rule Set 1.

- Dataset 2: a single-floor building model reconstructed based on Rule Set 2.

- Dataset 3: a multi-floor building model reconstructed based on Rule Set 3.

- Dataset 4: a multi-floor building model reconstructed based on Rule Set 4. where Rule Set 2 and Rule Set 4 include specific rules for selected building structures, while Rule Set 1 and Rule Set 3 only include the rules for general building structures. It is also noted that the comparison between reference model and test model in each dataset will be performed via visual inspection.

As shown in Figure 4, single-floor building models are produced from manual work, Rule Set 1 and Rule Set 2. The sub-figure 4a shows the manually processed model which is used as the reference, and the sub-figures $4 \mathrm{~b}$ and $4 \mathrm{c}$ represent the test models which are deemed as Dataset 1 and Dataset 2, respectively. In the test models, different colours are used to visualise the different building elements, such as roof element in blue, window element in green, wall column element in yellow, and base wall element in red. In sub-figures $4 \mathrm{~b}$, only determined model elements are displayed. Any missing parts are due to unknown objects detected e.g. roof element. It can be seen that this test model has significant differences with the reference model. In sub-figure $4 c$, a few errors have been marked out in blue circle. It is noted that the deficiency $a$ and $b$ are due to the errors brought from segmentation data, which could not be rectified with the existing grammar and rules. However, the semantic information of those roof element and wall element is determined correctly with the grammar method, which thereby gives out the expected colours. The deficiency $c$ is the problem caused by the wrong decision made by grammar engine in the reconstruction algorithm.

Similarly, Figure 5 shows the reference model and test models for the multi-floor building. Sub-figure $5 b$ shows the Dataset 3 , which fails to display all window frames as those segments cannot be determined by Rule Set 3. Also two base wall columns, bottom wall patches and door element are determined as other building elements incorrectly in this test model. For better Rule Set 4, sub-figure 5c indicates the test model as Dataset 4 . The deficiency $a$ is due to incorrect decision made by Rule Set 4, which has put the door element as a window element mistakenly.

\begin{tabular}{|c|c|c|c|c|c|c|}
\hline Dataset & $\rho_{d}$ & $\rho_{b}$ & $\rho_{q}$ & $\rho_{m}$ & $\rho_{f}$ & $\beta$ \\
\hline \hline 1 & 0.59 & 0.67 & 0.42 & 0.70 & 0.39 & 0.41 \\
\hline 2 & 0.95 & 0.06 & 0.90 & 0.06 & 0.05 & 0.05 \\
\hline 3 & 0.19 & 0.13 & 0.19 & 4.25 & 0.02 & 0.81 \\
\hline 4 & 0.99 & 0.01 & 0.99 & 0.01 & 0.01 & 0.01 \\
\hline
\end{tabular}

\begin{tabular}{|c|c|c|c|c|}
\hline Dataset & $|R|$ & $|D|$ & $|R \backslash D|$ & $|D \backslash R|$ \\
\hline \hline 1 & 56 & 55 & 23 & 22 \\
\hline 2 & 56 & 56 & 3 & 3 \\
\hline 3 & 168 & 36 & 136 & 4 \\
\hline 4 & 168 & 168 & 1 & 1 \\
\hline
\end{tabular}

Table 1: Quality measures and statistics for Dataset 1 - 4. D is denoted as the test dataset and $R$ is denoted as the reference dataset.

In order to evaluate entire building model in a quantitative way, Table 1 summarises the quantities and statistics based on six quality measures defined in 3.4. It is noted that dataset 2 has significant higher detection rate $\left(\rho_{d}\right)$ and quality rate $\left(\rho_{q}\right)$, but much lower branch factor $\left(\rho_{b}\right)$, miss factor $\left(\rho_{m}\right)$, false alarm rate $\left(\rho_{f}\right)$ and type 2 error $(\beta)$. Dataset 2 is much closer to the optimised result compared with dataset 1 . The same trend is observed between dataset 4 and dataset 3 . It is obvious that dataset 4 has better quality measurement results compared with dataset 3 . The quantitative analysis from introduced quality 


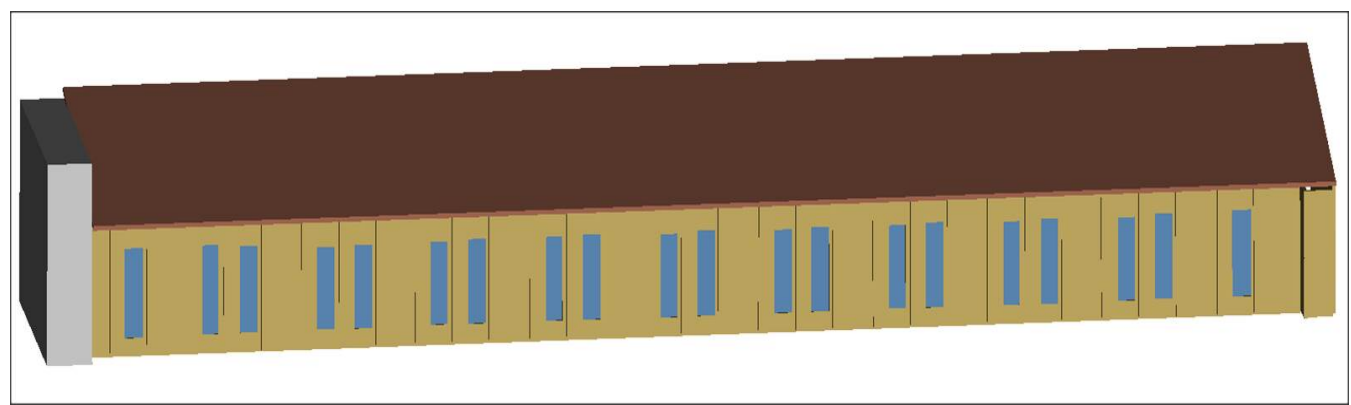

(a) Manual reconstruction of a single-floor building model.

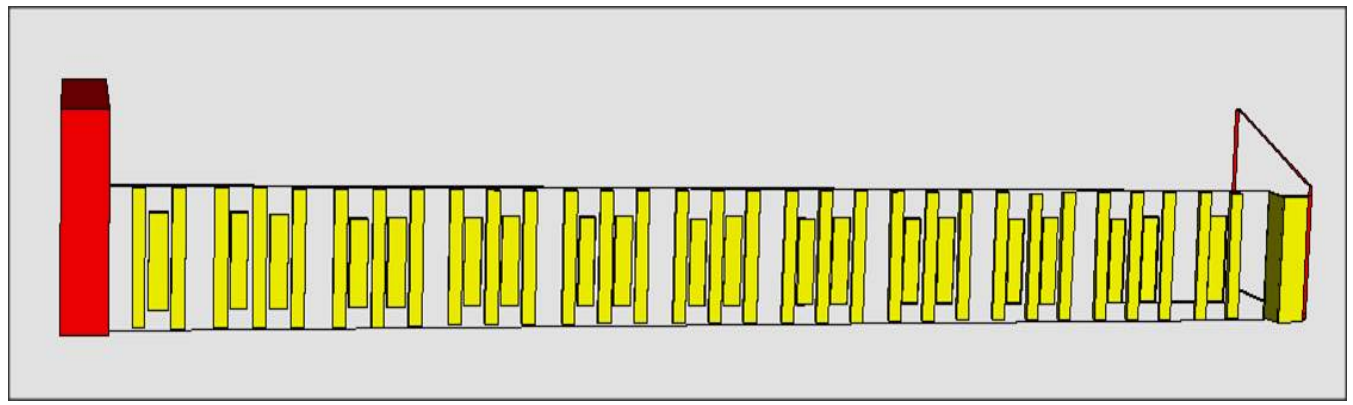

(b) Automatic reconstruction of a single-floor building model with Rule Set 1. Only determined model elements are displayed.

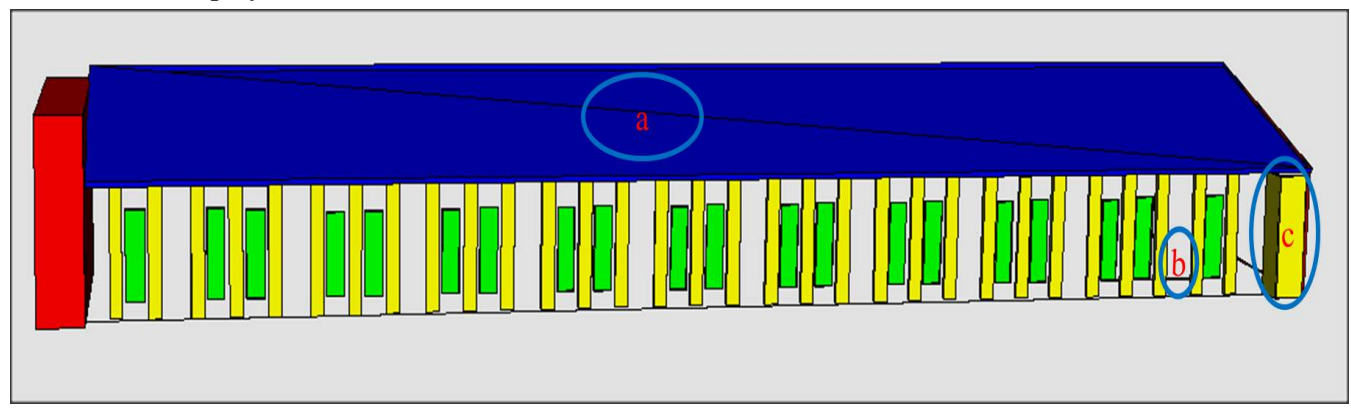

(c) Automatic reconstruction of a single-floor building model with Rule Set 2. Compared with the reference model, the errors are marked out in blue circles.

Figure 4: Comparison of manual processed model and grammar-based automatic reconstructed models with different rule sets for a single-floor building. Colours in reconstructed models stand for roof in blue, window in green, wall column in yellow, and base wall in red.

measures is consistent with the results of Figure 4 and Figure 5, which proves the proposed evaluation method is working.

\section{CONCLUSIONS}

The objective of this paper is to propose a quantitative quality evaluation method to assess the completeness and correctness of 3D building model reconstructed from a grammar-based approach. For this purpose, six quality measures have been introduced for the evaluation of a 3D building model mainly from the perspective of semantic information, which are detection rate $\left(\rho_{d}\right)$, branch factor $\left(\rho_{b}\right)$, quality rate $\left(\rho_{q}\right)$, miss factor $\left(\rho_{m}\right)$, false alarm rate $\left(\rho_{f}\right)$ and type 2 error $(\beta)$, respectively. The reconstructed $3 \mathrm{D}$ building model is compared to the reference building model from second independent compilation, e.g. manual process. Differences between the test and the reference models are inspected through visualisation to calculate the criteria values, which determine the overall quality of the reconstructed models. By using various grammar rule sets, the test model datasets are varied accordingly. It has been proved that the proposed quality measures have the correct indications to evaluate the reconstructed building model.
In future work, additional 2D evaluation for building area based on existing available 2D floor plan information is required to combine with the proposed method, which will provide more accurate quality evaluation of full 3D building models.

\section{ACKNOWLEDGEMENTS}

The work has been supported by the Cooperative Research Centre for Spatial Information (CRCSI), whose activities are funded by the Australian Commonwealth's Cooperative Research Centres Programme. This work has been permitted for publication under CRCSI publication policy.

\section{References}

Akca, D., Freeman, M., Sargent, I. and Gruen, A., 2010. Quality assessment of $3 \mathrm{~d}$ building data. The Photogrammetric Record 25(132), pp. 339-355.

Ameri, B., 2000. Feature-based model verification (fbmv): a new concept for hypothesis validation in building reconstruction. 


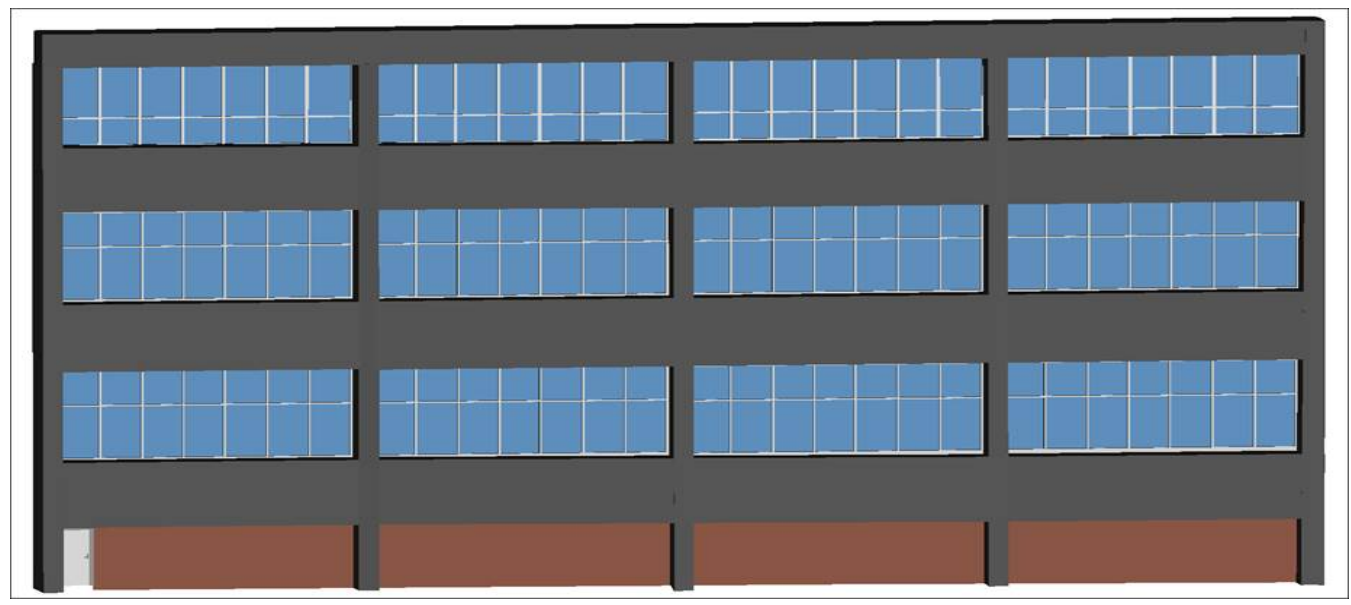

(a) Manual reconstruction of a multi-floor building model.

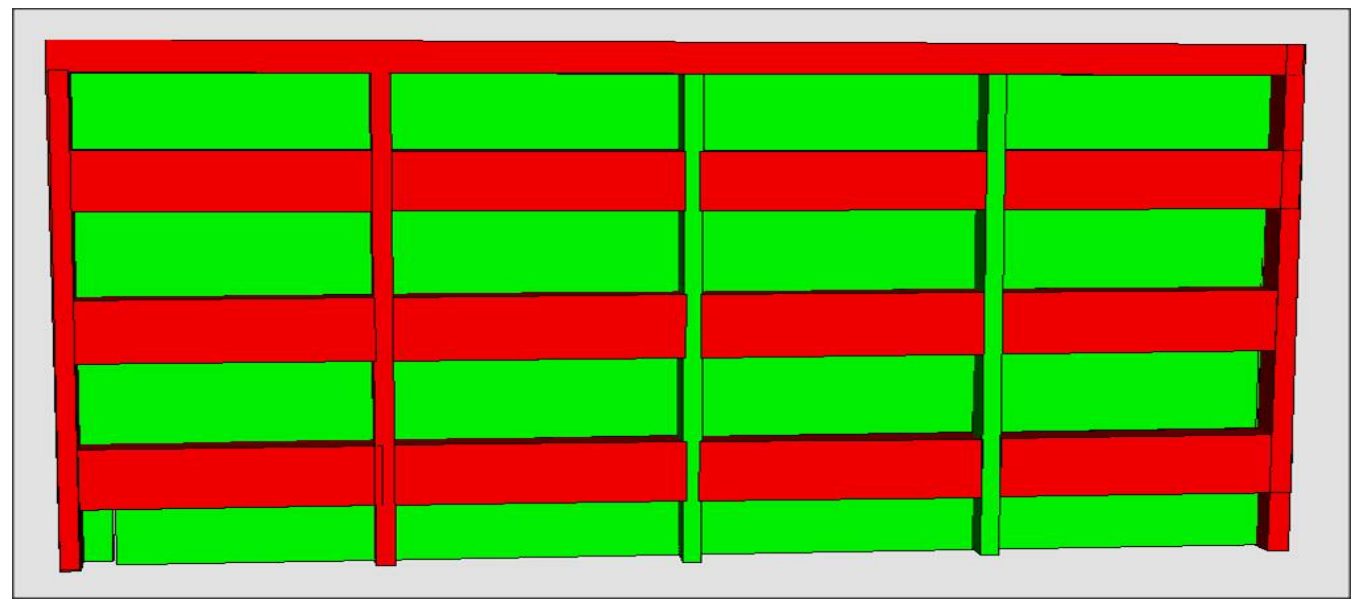

(b) Automatic reconstruction of a multi-floor building model with Rule Set 3. Only determined model elements are displayed.

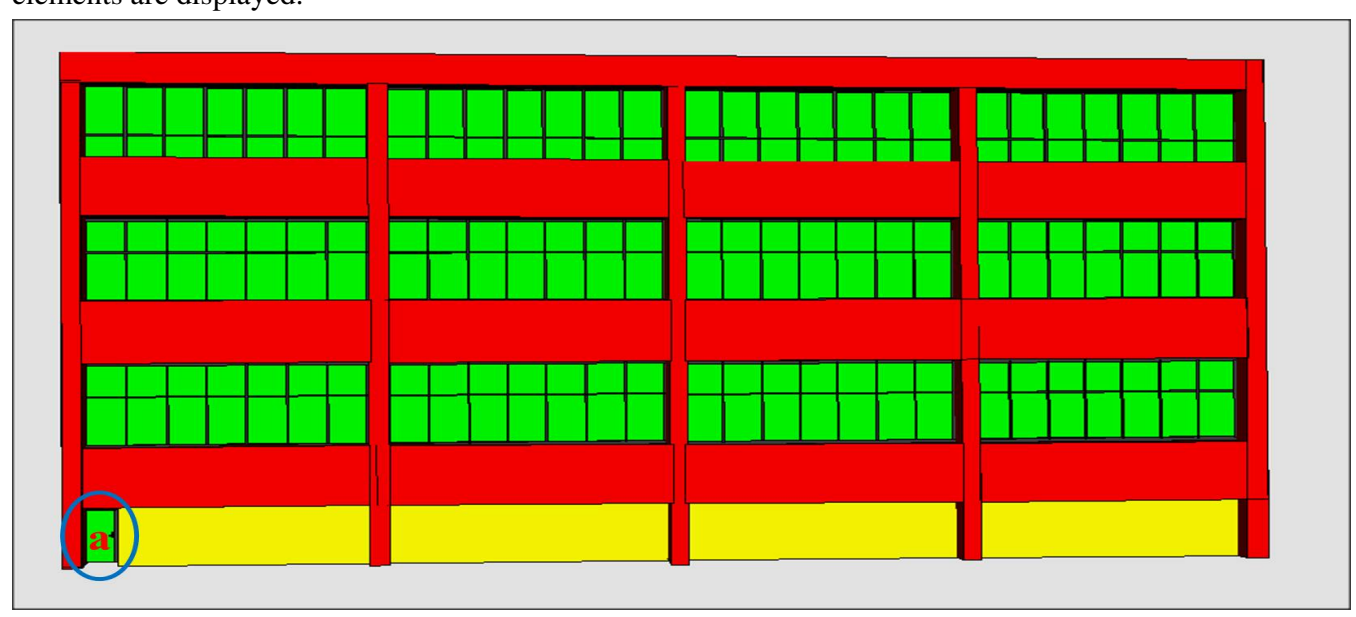

(c) Automatic reconstruction of a multi-floor building model with Rule Set 4. Compared with the reference model, the error is marked out in blue circle.

Figure 5: Comparison of manual processed model and grammar-based automatic reconstructed models with different rule sets for a multi-floor building. Colours in the reconstructed models stand for window in green, wall patch in yellow, and base wall in red.

In: Proceedings of the XIXth ISPRS Congress, IAPRS XIX, Part 3, pp. 24-35.

Becker, S., 2009. Generation and application of rules for quality dependent facade reconstruction. ISPRS Journal of Photogrammetry and Remote Sensing 64(6), pp. 640-653.

Becker, S. and Haala, N., 2009. Grammar supported facade reconstruction from mobile lidar mapping. ISPRS Workshop, CMRT09 - City Models, Roads and Traffic (2009) pp. 229-234.

Biljecki, F., Zhao, J., Stoter, J. and Ledoux, H., 2013. Revisiting the concept of level of detail in $3 \mathrm{~d}$ city modelling. ISPRS Annals of Photogrammetry, Remote Sensing and Spatial Information Sciences II-2/W1, pp. 63-74. 
Brenner, C., 2005. Building reconstruction from images and laser scanning. International Journal of Applied Earth Observation and Geoinformation 6(34), pp. 187 - 198.

Dorninger, P. and Pfeifer, N., 2008. A comprehensive automated $3 \mathrm{~d}$ approach for building extraction, reconstruction, and regularization from airborne laser scanning point clouds. Sensors 8(11), pp. 7323.

Durupt, M. and Taillandier, F., 2006. Automatic building reconstruction from a digital elevation model and cadastral data: an operational approach. Proc. of the ISPRS Commission III Symposium on Photogrammetric and Computer Vision, Bonn, Germany (on CD-ROM).

Elberink, S. O. and Vosselman, G., 2009. Building reconstruction by target based graph matching on incomplete laser data: Analysis and limitations. Sensors 9(8), pp. 6101.

Elberink, S. O. and Vosselman, G., 2011. Quality analysis on $3 \mathrm{~d}$ building models reconstructed from airborne laser scanning data. ISPRS Journal of Photogrammetry and Remote Sensing 66(2), pp. 157-165.

Haala, N. and Kada, M., 2010. An update on automatic 3d building reconstruction. ISPRS Journal of Photogrammetry and Remote Sensing 65(6), pp. 570 - 580.

Helmholz, P., , Belton, D. and Moncrieff, S., 2013. Approach for the semi-automatic verification of $3 \mathrm{~d}$ building models. ISPRS - International Archives of the Photogrammetry, Remote Sensing and Spatial Information Sciences XL-1/W1, pp. 121126.

Henricsson, O. and Baltsavias, E., 1997. 3d building reconstruction with aruba: A qualitative and quantitative evaluation. Int. Workshops "Automatic Extraction of ManMade Objects from Aerial and Space Images (II)” pp. 65-76.

McGlone, J. C. and Shufelt, J. A., 1994. Projective and object space geometry for monocular building extraction. Conference on Computer Vision and Pattern Recognition, CVPR 1994, Seattle, WA, USA pp. 54-61.

McKay, A., Chase, S. C., Shea, K. and Chau, H. H., 2012. Spatial grammar implementation: From theory to useable software. AI EDAM (Artificial Intelligence for Engineering Design, Analysis and Manufacturing) 26, pp. 143-159.

McKeown, D. M., Bulwinkle, T., Cochran, S., Harvey, W., McGlone, C. and Shufelt, J. A., 2000. Performance evaluation for automatic feature extraction. IAPRS 33 (B2), pp. 379-394.

Milde, J., Zhang, Y., Brenner, C., Plümer, L. and Sester, M., 2008. Building reconstruction using a stuctural description based on a formal grammar. ISPRS - International Archives of the Photogrammetry, Remote Sensing and Spatial Information Sciences III-3, pp. 227-232.

Moreira, J. M., Nex, F., Agugiaro, G., Remondino, F. and Lim, N., 2013. From dsm to 3d building models: A quantitative evaluation. ISPRS - International Archives of the Photogrammetry, Remote Sensing and Spatial Information Sciences XL-1/W1, pp. 213-219.

Müller, P., Wonka, P., Haegler, S., Ulmer, A. and Gool, L. V., 2006. Procedural modeling of buildings. Proceedings of ACM SIGGRAPH 2006 / ACM Transactions on Graphics 25(3), pp. 614-623.
Nurunnabi, A., Belton, D. and West, G., 2012. Robust segmentation in laser scanning $3 \mathrm{~d}$ point cloud data. Digital Image Computing Techniques and Applications (DICTA), 2012 pp. 1-8.

Parish, Y. I. H. and Müller, P., 2001. Procedural modeling of cities. Proceedings of the 28th Annual Conference on Computer Graphics and Interactive Techniques pp. 301-308.

Rabin, S., 2002. AI Game Programming Wisdom. Charles River Media, Inc., Rockland, MA, USA.

Ragia, L., 2000. A quality model for spatial objects. Proceedings of the 19th ISPRS Congress pp. 855-862, 4C.

Ripperda, N. and Brenner, C., 2009. Evaluation of structure recognition using labelled facade images. Proceedings of the 31 st DAGM Symposium on Pattern Recognition pp. 532-541.

Rottensteiner, F. and Schulze, M., 2003. Performance evaluation of a system for semi-automatic building extraction using adaptable primitives. ISPRS Archives XXXIV-3/W8, pp. 4752.

Schuster, H.-F. and Weidner, U., 2003. A new approach towards quantitative quality evaluation of $3 \mathrm{~d}$ building models. ISPRS Comm. IV Joint Workshop Challenges in Geospatial Analysis" pp. 8-9.

Stiny, G. and Gips, J., 1972. Shape grammars and the generative specification of painting and sculpture. Information Processing 71 pp. $1460-1465$.

Suveg, I. and Vosselman, G., 2002. Mutual information based evaluation of $3 \mathrm{~d}$ building models. 16th International Conference on Pattern Recognition, ICPR, Quebec, Canada, 2002 vol 3, pp. 557-560.

Vosselman, G. and Maas, H.-G., 2010. Airborne and Terrestrial Laser Scanning. Whittles Publishing.

Wonka, P., Wimmer, M., Sillion, F. and Ribarsky, W., 2003. Instant architecture. ACM Trans. Graph. 22(3), pp. 669-677.

Yu, Q., Helmholz, P., Belton, D. and West, G., 2014. Grammarbased automatic $3 \mathrm{~d}$ model reconstruction from terrestrial laser scanning data. ISPRS - International Archives of the Photogrammetry, Remote Sensing and Spatial Information Sciences XL-4, pp. 335-340.

Yu, Q., Helmholz, P., Belton, D. and West, G., 2015. Semantically enhanced $3 \mathrm{~d}$ building model reconstruction from terrestrial laser scanning data. Presented at The 9th International Symposium on Mobile Mapping Technology MMT 2015, 9-11 Dec. Sydeney. 